\title{
Current status and dilemma of second-line treatment in advanced pancreatic cancer: is there a silver lining?
}

Jie Hua ${ }^{1-4, *}$

Si Shi ${ }^{1-4, *}$

Dingkong Liangl-4

Chen Liangl-4

Qingcai Mengl-4

Bo Zhangl-4

Quanxing $\mathrm{Ni}^{1-4}$

Jin $X u^{1-4}$

Xianjun $\mathrm{Yu}^{1-4}$

'Department of Pancreatic Surgery,

Fudan University Shanghai Cancer

Center, Shanghai, People's Republic

of China; ${ }^{2}$ Department of Oncology,

Shanghai Medical College, Fudan

University, Shanghai, People's

Republic of China; ${ }^{3}$ Pancreatic Cancer Institute, Fudan University, Shanghai,

People's Republic of China; ${ }^{4}$ Shanghai

Pancreatic Cancer Institute, Shanghai,

People's Republic of China

*These authors contributed equally to this work
This article was published in the following Dove Press journal:

OncoTargets and Therapy

\begin{abstract}
Pancreatic cancer remains one of the most lethal malignant diseases worldwide. The majority of patients present with advanced disease and, therefore, need palliative chemotherapy. Some chemotherapeutic regimens have been well established as first-line therapies and have been shown to increase survival; however, almost all patients with advanced pancreatic cancer will experience disease progression after first-line therapy. Nevertheless, many patients who retain good performance status after initial treatment remain good candidates for additional therapy. Historically, few studies have assessed second-line therapy, with most reports representing small phase II trials with variable findings; however, clinical research for second-line treatment has increased in the past decade, and several randomized controlled trials using different regimens have been published. The current literature shows varying results on treatment efficacy and tolerability. Thus, we reviewed the published data on the use of chemotherapy in the second-line setting for the treatment of advanced pancreatic cancer.
\end{abstract}

Keywords: pancreatic cancer, second-line, chemotherapy, targeted therapy

\section{Introduction}

Pancreatic cancer is a highly lethal disease, for which the 5-year relative survival rate is approximately $7 \%-8 \%{ }^{1,2}$ It remains the fourth leading cause of cancer-related deaths, with approximately 43,090 deaths estimated in the US in $2017 .{ }^{3}$ Most patients (approximately 80\%) are ineligible for potential curative resection at diagnosis. ${ }^{4}$ In these cases, pancreatic cancer is diagnosed at an advanced stage - either as locally advanced pancreatic cancer (LAPC) or as metastatic disease - with a median overall survival (OS) of 9-15 and 6-9 months, respectively. ${ }^{5}$ Gemcitabine has been the standard first-line treatment in this advanced setting for over a decade. ${ }^{6}$ However, since 2011, new chemotherapeutic regimens (FOLFIRINOX and nab-paclitaxel plus gemcitabine) have shown improved survival benefit over gemcitabine monotherapy and are the most commonly administered first-line therapies in patients with good performance status. ${ }^{7,8}$ Therefore, gemcitabine monotherapy is now more frequently used as an option for patients with advanced pancreatic cancer who have poor performance status. Nonetheless, almost all patients will inevitably experience disease progression during or following first-line therapy and, beyond first-line treatment, approximately half of all patients with advanced pancreatic cancer remain in good general condition and, therefore, may receive subsequent therapies. ${ }^{9}$

Historically, multiple phase II studies have used various single agents or combination regimens in the second-line setting to improve survival, although most 
of these attempts have been in vain. However, in recent years, second-line chemotherapies have been developed using different regimens as standards. Several randomized phase III trials, including NAPOLI-1, CONKO-003, and PANCREOX, have shown promising results in improving survival, thus representing potential second-line options for patients with gemcitabine-resistant pancreatic cancer. ${ }^{10-12}$ However, reflecting the contradictory results on treatment efficacy and tolerability and criticisms on selective enrollment and study biases, the optimal second-line strategy for pancreatic cancer remains unknown.

In this review, we summarize current second-line chemotherapy options, second-line targeted therapies, and the most promising second-line agents to highlight state-of-the-art and future directions of second-line treatment in advanced pancreatic cancer.

\section{Search strategy and selection criteria}

The data for this review were identified through searches of PubMed and references from relevant articles published in the English language using the following search terms: "advanced pancreatic cancer", “inoperable pancreatic adenocarcinoma", "gemcitabine-refractory pancreatic cancer", "second-line therapy", "second-line treatment", and "second-line chemotherapy". Abstracts from the annual meetings of the American Society of Clinical Oncology and European Society of Medical Oncology were also screened for additional studies. Only prospective clinical trials published between 1997 and 2017 were included. Studies were also required to meet the inclusion criteria that the first-line therapy was either a gemcitabine-based regimen (gemcitabine monotherapy or gemcitabine-containing combination therapy) or FOLFIRINOX. Abstracts and reports from meetings were included only when they directly associated with previously published work. The PRISMA (Preferred Reporting Items for Systematic Review and Meta-Analysis) flow diagram for this review is shown in Figure 1.

\section{Second-line chemotherapy in advanced pancreatic cancer: yes or no?}

Does the benefit of chemotherapy continue with disease progression during or after first-line treatment? Two randomized phase III trials compared second-line treatments with best supportive care (BSC). In the first study by Ciuleanu et al, 303 patients with previously gemcitabine-treated metastatic pancreatic adenocarcinoma were randomized to glufosfamide or BSC. ${ }^{13}$ The median survival was 105 days (3.5 months) for glufosfamide and 84 days (2.8 months) for BSC, with an 18\% increase in OS for glufosfamide, which was not statistically significant (HR 0.85; 95\% CI 0.66-1.08; $P=0.19$ ), suggesting low activity of glufosfamide in this refractory patient population. The second study by the German CONKO-study group randomized patients in a 1:1 ratio to oxaliplatin plus folinic acid and 5-fluorouracil (OFF) or BSC. ${ }^{14}$ Calculations were undertaken with a total of 165 patients to demonstrate a doubling of survival time after progression on first-line gemcitabine therapy. However, after inclusion of 46 patients (23 in each arm), the trial was terminated early due to insufficient accrual (lack of acceptance of BSC by patients and physicians). Although no confirmed response better than stable disease was observed, OFF as second-line chemotherapy significantly prolonged survival time compared to BSC alone (4.82 vs 2.30 months, $P=0.008$; Table 1).

These two studies did not yield sufficient evidence to demonstrate the necessity of second-line chemotherapy (partly because one study used glufosfamide, which was not commonly used in the treatment of pancreatic cancer, and the other study was prematurely closed); however, this treatment may have a survival benefit in this patient population. Further trials in the second-line setting are needed, comparing active treatment with $\mathrm{BSC}$ to ensure that patients do not receive unnecessary toxicity without any benefit. That is, from an ethical perspective, patient-centered decision-making becomes the highest priority. Therefore, a comprehensive assessment considering performance status, comorbidities, expected toxicities, cost-effectiveness, and patient preference is required.

\section{Second-line single-agent chemotherapy: one is all?}

A summary of the prospective clinical trials on second-line single-agent chemotherapy is shown in Table 2. All of these trials were phase II studies. Although few patients showed an objective response (range $0-15 \%$ ), the disease control rate ranged from $16.7 \%$ to $58 \%$, and the median second-line survival ranged from 3.5 to 7.6 months.

Based on the data of increased cytotoxic activity of oxaliplatin in pancreatic cell lines with microsatellite instability, Androulakis et al conducted a phase II trial that enrolled 18 advanced pancreatic cancer patients with a previous history of gemcitabine-based chemotherapy treatment. ${ }^{15}$ Oxaliplatin was administered at $130 \mathrm{mg} / \mathrm{m}^{2}$ every 3 weeks. The study failed to induce an objective response, and three patients had stable disease for over 2 months. Hematological toxicity was mild, with no grade 3 or 4 toxic effects reported. 


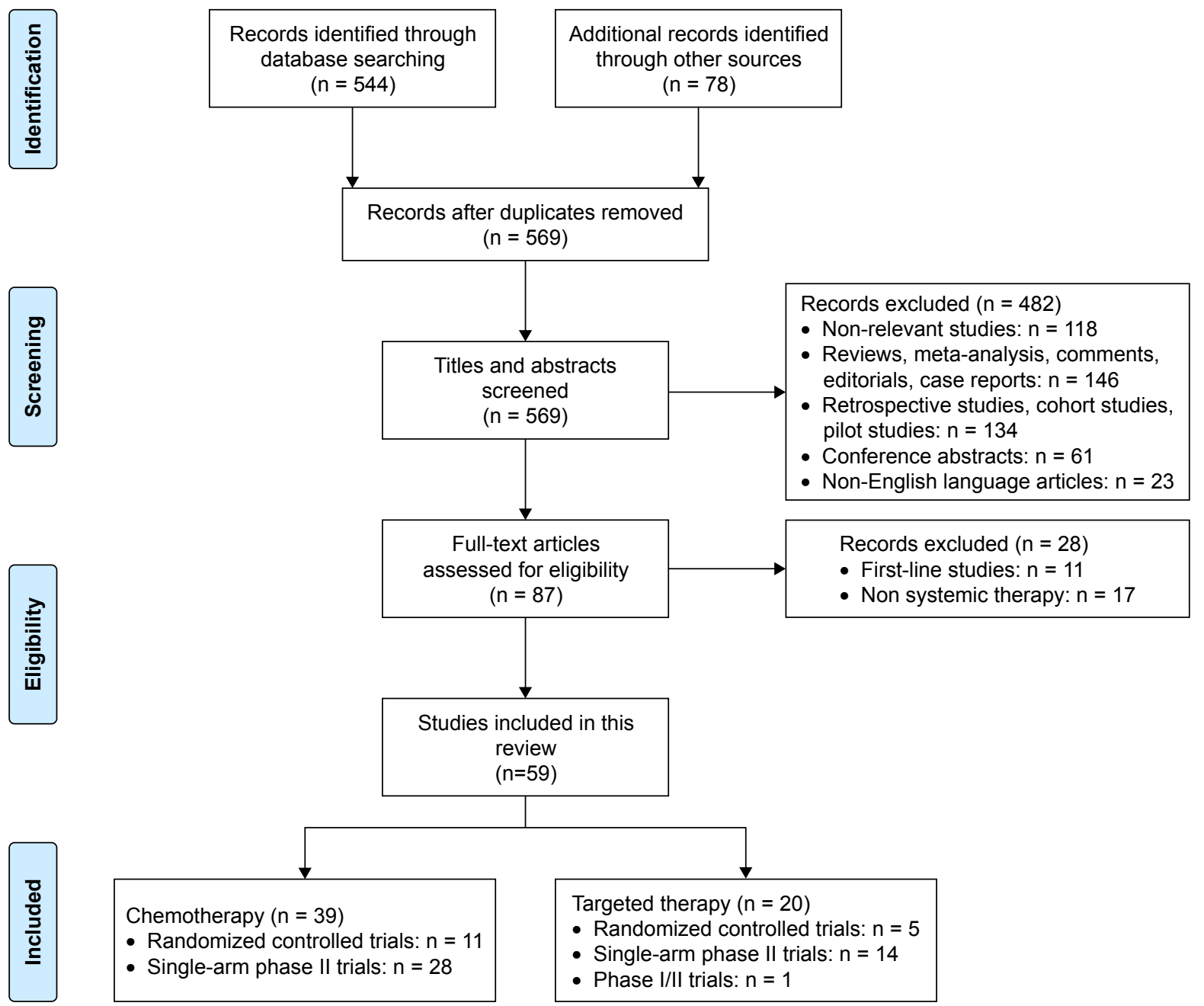

Figure I PRISMA (Preferred Reporting Items for Systematic Review and Meta-Analysis) flow diagram of study selection.

In patients with advanced pancreatic cancer, first-line chemotherapy with pemetrexed has shown clinical activity as a single agent as well as in combination with gemcitabine. Based on this result, a multicenter phase II trial was conducted to evaluate the efficacy and safety of pemetrexed as a second-line treatment in patients with advanced pancreatic cancer who had progressed on single-agent gemcitabine or gemcitabine-based first-line chemotherapy. ${ }^{16} \mathrm{~A}$ total of 52 patients received pemetrexed at $500 \mathrm{mg} / \mathrm{m}^{2}$ intravenously every 3 weeks. The response rate was $3.8 \%(95 \%$ CI $0.5 \%-13.2 \%)$. At a median follow-up of 20 weeks, the median time to progression (TTP) was 7 weeks, and the median OS was 20 weeks. The most frequent hematological grades 3 and 4 toxic effects included neutropenia (17.3\%), thrombocytopenia (5.8\%), and anemia (3.8\%). The most frequent non-hematological toxic effects (any grade) were diarrhea, nausea, and stomatitis/pharyngitis (23.1\% each). The authors concluded that pemetrexed is a safe treatment option with limited activity in the second-line setting after gemcitabine failure.

Second-line chemotherapy with single-agent tegafur/ gimeracil/oteracil (S-1) in patients with gemcitabine-resistant advanced pancreatic cancer has also been assessed. Morizane et al examined S- 1 treatment at $40 \mathrm{mg} / \mathrm{m}^{2}$ twice a day for 4 weeks on a 6-week cycle and showed that the response rate was $15 \%$ with a median progression-free survival (PFS) of 2.0 months and a median OS of 4.5 months. ${ }^{17}$ The toxicity was tolerable, and no life-threatening toxicities were observed. Another study using the same regimen showed a similar response rate of $9.5 \%$, and PFS and OS were 4.1 and 6.3 months, respectively. ${ }^{18}$ Moreover, the toxicity profile was similar.

Irinotecan monotherapy has been tested in patients with previously untreated advanced pancreatic cancer, and the activity observed with irinotecan was at least equivalent to, if not better than, that of gemcitabine. Based on these data, Yi et al initiated a phase II study of single-agent irinotecan 


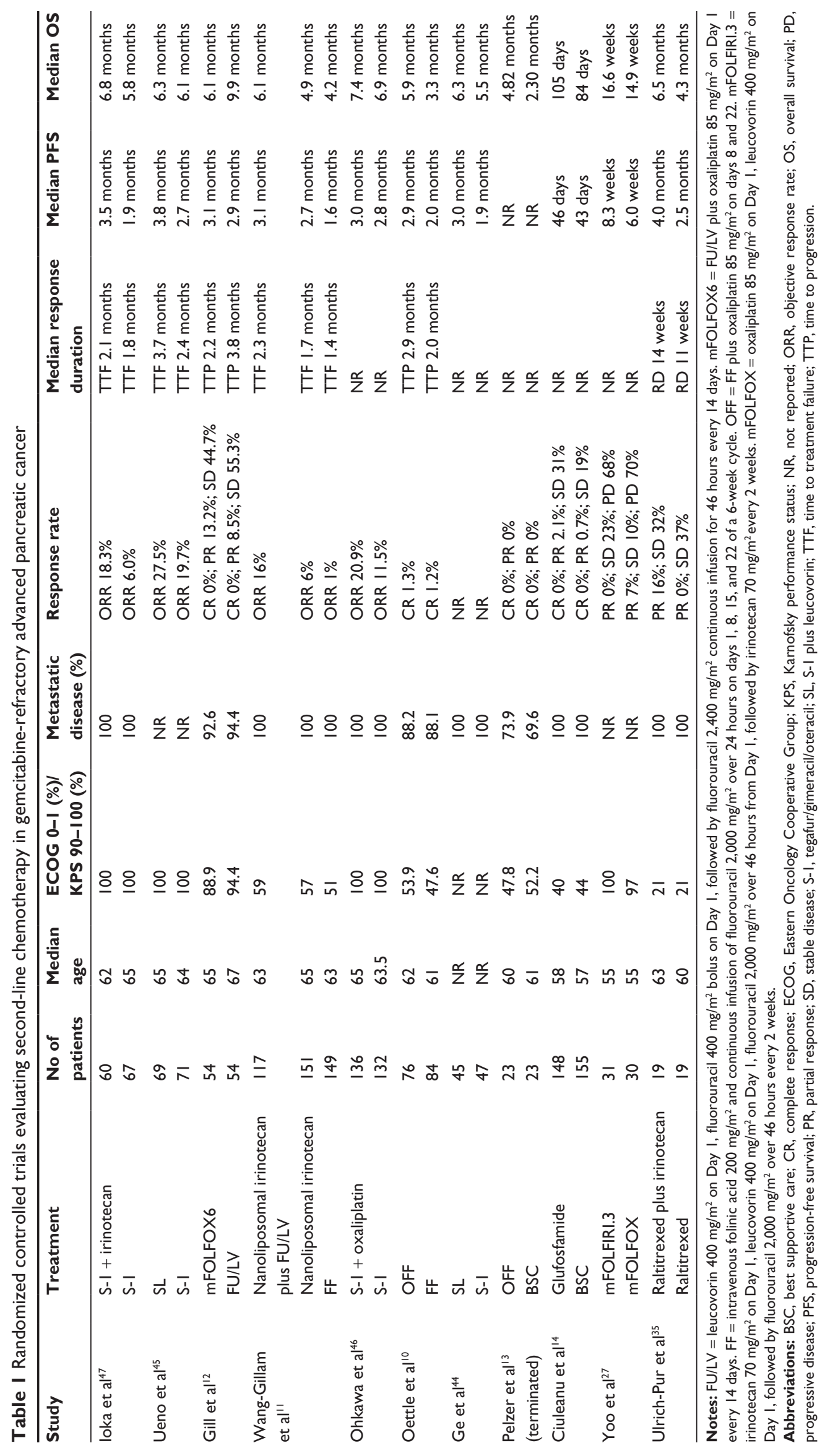


Table 2 Single-armed phase II clinical trials evaluating second-line single-agent chemotherapy in advanced pancreatic cancer

\begin{tabular}{|c|c|c|c|c|c|c|}
\hline Study & Treatment & $\begin{array}{l}\text { No of } \\
\text { patients* }\end{array}$ & $\begin{array}{l}\text { ECOG 0-I (\%)/ } \\
\text { KPS } 90-100(\%)\end{array}$ & $\begin{array}{l}\text { Metastatic } \\
\text { disease (\%) }\end{array}$ & Response rate & $\begin{array}{l}\text { Median } \\
\text { survival }\end{array}$ \\
\hline Hosein et $\mathrm{a}^{22}$ & $\begin{array}{l}\text { Nab-paclitaxel } 100 \mathrm{mg} / \mathrm{m}^{2} \text { on days I, } 8 \text {, } \\
\text { and } 15 \text { of a } 28 \text {-day cycle }\end{array}$ & 19 & 79 & 95 & $\begin{array}{l}\text { CR 0\%; PR 5\%; } \\
\text { SD 32\% }\end{array}$ & $\begin{array}{l}\text { OS } 7.3 \text { months; } \\
\text { PFS I.7 months }\end{array}$ \\
\hline Ko et $\mathrm{a}^{20}$ & $\begin{array}{l}\text { Nanoliposomal irinotecan } 120 \mathrm{mg} / \mathrm{m}^{2} \\
\text { every } 3 \text { weeks }\end{array}$ & 40 & 60 & 100 & $\begin{array}{l}\text { PR 7.5\%; SD 42.5\%; } \\
\text { PD } 25.0 \%\end{array}$ & $\begin{array}{l}\text { OS } 5.2 \text { months; } \\
\text { PFS } 2.4 \text { months }\end{array}$ \\
\hline Sudo et al ${ }^{18}$ & $\begin{array}{l}\text { S-I } 40 \mathrm{mg} / \mathrm{m}^{2} \text { orally, twice a day for } \\
4 \text { weeks on a 6-week cycle }\end{array}$ & 21 & 71.4 & 95 & PR 9.5\%; SD 43\% & $\begin{array}{l}\text { OS } 6.3 \text { months; } \\
\text { PFS } 4.1 \text { months }\end{array}$ \\
\hline Yi et al ${ }^{19}$ & Irinotecan $150 \mathrm{mg} / \mathrm{m}^{2}$ every 2 weeks & 33 & 94 & 100 & PR 9\%; SD 39\% & $\begin{array}{l}\text { OS } 6.6 \text { months; } \\
\text { PFS } 2.0 \text { months }\end{array}$ \\
\hline Morizane et al ${ }^{17}$ & $\begin{array}{l}\text { S-I } 40 \mathrm{mg} / \mathrm{m}^{2} \text { orally, twice a day for } \\
4 \text { weeks on a 6-week cycle }\end{array}$ & 40 & 90 & 100 & $\begin{array}{l}\text { CR 0\%; PR I5\%; } \\
\text { SD 43\%; PD 38\% }\end{array}$ & $\begin{array}{l}\text { OS } 4.5 \text { months; } \\
\text { PFS } 2.0 \text { months }\end{array}$ \\
\hline Boeck et $\mathrm{al}^{21}$ & $\begin{array}{l}\text { Capecitabine } 1,250 \mathrm{mg} / \mathrm{m}^{2} \text { orally, twice } \\
\text { a day for } 2 \text { weeks on a 3-week cycle }\end{array}$ & 39 & 51 & 97 & $\begin{array}{l}\text { PR 0; SD 39\%; } \\
\text { PD } 31 \%\end{array}$ & OS 7.6 months \\
\hline Boeck et al ${ }^{16}$ & Pemetrexed $500 \mathrm{mg} / \mathrm{m}^{2}$ every 3 weeks & 52 & 94.2 & 88.5 & $\begin{array}{l}\text { PR 3.8\%; SD 19.2\%; } \\
\text { PD 59.6\% }\end{array}$ & OS 20 weeks \\
\hline Androulakis et al ${ }^{15}$ & Oxaliplatin $130 \mathrm{mg} / \mathrm{m}^{2}$ every 3 weeks & 18 & 72 & 72 & $\begin{array}{l}\text { CR 0\%; PR 0\%; } \\
\text { SD } 16.7 \%\end{array}$ & OS 3.5 months \\
\hline
\end{tabular}

Notes: *All patients were previously treated with first-line gemcitabine monotherapy or gemcitabine-based combination therapy.

Abbreviations: CR, complete response; ECOG, Eastern Oncology Cooperative Group; KPS, Karnofsky performance status; OS, overall survival; PD, progressive disease; PFS, progression-free survival; PR, partial response; SD, stable disease; S-I, tegafur/gimeracil/oteracil.

as second-line chemotherapy in patients with advanced pancreatic cancer. ${ }^{19}$ Among 33 patients, three patients showed a partial response, and 13 patients with stable disease were observed. The median PFS and OS were 2.0 and 6.6 months, respectively. Toxicity profiles were generally manageable, and there was no treatment-related death. Nanoliposomal irinotecan is a liposomal nanoparticle that encapsulates free irinotecan. This structure protects the irinotecan base from being converted to its active metabolite, SN-38, which enables a longer duration of the drug in circulation - thus increasing and prolonging the intratumoral levels of irinotecan and SN-38. In a phase II study of 40 patients with metastatic pancreatic ductal adenocarcinoma previously treated with gemcitabine-based therapy, the administration of nanoliposomal irinotecan at $120 \mathrm{mg} / \mathrm{m}^{2}$ every 3 weeks resulted in a median OS of 5.2 months, 1-year survival of $25 \%$, and a manageable toxicity profile. ${ }^{20}$

Capecitabine, a prodrug of fluorouracil, has shown single-agent activity in the first-line treatment of patients with advanced pancreatic cancer. Boeck et al also examined capecitabine as salvage chemotherapy in gemcitabinepretreated patients. ${ }^{21}$ This agent was orally administered to 39 patients at a dose of $1,250 \mathrm{mg} / \mathrm{m}^{2}$ twice daily for 14 days, followed by 7 days of rest. After a median follow-up of 6.6 months, 27 patients were evaluated for their response: no complete or partial responses were observed, but 15 patients (39\%) had stable disease. The median TTP was 2.3 months, and the median OS was 7.6 months. Predominant grades 2 and 3 toxicities included hand-foot syndrome (28\%), anemia
(23\%), leg edema (15\%), diarrhea (13\%), nausea/vomiting $(10 \%)$, and leukocytopenia (10\%).

Nab-paclitaxel - which can breach the blood-stroma barrier to reach tumor cells - was also tested in the secondline setting for advanced pancreatic cancer prior to the phase III MAPCT trial. ${ }^{22}$ Patients received nab-paclitaxel at $100 \mathrm{mg} / \mathrm{m}^{2}$ for over 30 minutes on days 1,8 , and 15 of a 28-day cycle. Among the 19 treated patients, one patient had a confirmed partial response and 6 patients had stable disease as their best response. The median PFS and OS were 1.7 and 7.3 months, respectively. Grades 3 and 4 neutropenia, neutropenic fever, and anemia occurred in $32 \%, 11 \%$, and $11 \%$ of patients, respectively.

In summary, single-agent chemotherapy may constitute a feasible treatment option with acceptable activity and tolerable toxicity for patients with gemcitabine-refractory pancreatic cancer. In view of the favorable toxicity profile, single agents combined with other agents might improve the therapeutic results.

\section{Second-line combination chemotherapy: it takes two to tango?}

As a patient's performance status often rapidly declines when the tumor is locally or systemically progressing, it can be difficult to administer second-line combination chemotherapy in pancreatic cancer. However, it is reasonable to consider salvage chemotherapy in a selective patient population. Several clinical trials have evaluated the efficacy and safety of different combination regimens in patients with advanced 
pancreatic cancer previously treated with gemcitabine-based therapies, with some combinations yielding promising results (Tables 1 and 3).

\section{Platinum-based regimens}

Many studies have used oxaliplatin as part of a combination regimen - the most commonly used being oxaliplatin plus infusional fluorouracil. The OFF regimen administered on a 6-week cycle was feasible and active, with an acceptable toxicity profile. ${ }^{23}$ As discussed earlier, the OFF regimen as second-line therapy showed a survival benefit compared with BSC in gemcitabine-refractory disease. ${ }^{14}$ The German CONKO-study group further conducted a randomized phase III trial comparing OFF to folinic acid and fluorouracil (FF) and showed that the OFF regimen was associated with a significantly improved OS (5.9 vs 3.3 months; $P=0.010)$ and PFS (2.9 vs 2.0 months; $P=0.019) .{ }^{10}$ The addition of oxaliplatin to FF increased neurotoxicity (mostly, grades 1 and 2) but was well tolerated. However, the results of a more recent randomized phase III trial (PANCREOX) using a biweekly infusional fluorouracil, leucovorin, and oxaliplatin (FOLFOX) schedule were disappointing, with similar PFS (3.1 vs 2.9 months; $P=0.99$ ) and shorter OS (6.1 vs 9.9 months; $P=0.02$ ) in the modified FOLFOX6 group versus the infusional fluorouracil/leucovorin (FU/LV) group. ${ }^{12}$ Moreover, the response rates were not significantly different between groups $(13.2 \%$ vs $8.5 \% ; P=0.361)$. The tolerability of the infusional FU/LV group was remarkably better than that of the modified FOLFOX6 group, with a nearly six-fold lower incidence of grades $3 / 4$ adverse events (11\% vs 63\%).

Other oxaliplatin-fluoropyrimidine combinations have also been evaluated in small phase II studies. In a comparable regimen to OFF, Tsavaris et al used weekly $50 \mathrm{mg} / \mathrm{m}^{2}$ oxaliplatin, $50 \mathrm{mg} / \mathrm{m}^{2}$ leucovorin, and $500 \mathrm{mg} / \mathrm{m}^{2}$ fluorouracil treatments and achieved a median OS of 25 weeks. ${ }^{24}$ Novarino et al administered $40 \mathrm{mg} / \mathrm{m}^{2}$ oxaliplatin, $250 \mathrm{mg} / \mathrm{m}^{2}$ leucovorin, and $500 \mathrm{mg} / \mathrm{m}^{2}$ fluorouracil weekly for a treatment period of 3 weeks on a 4-week cycle, resulting in a median OS of 17.1 weeks. ${ }^{25}$ In a similar study conducted by El-Hadaad and Wahba, the median OS was 22 weeks. ${ }^{26}$ Additionally, a small phase II trial in Korea randomly assigned patients to receive $85 \mathrm{mg} / \mathrm{m}^{2}$ oxaliplatin, $400 \mathrm{mg} / \mathrm{m}^{2}$ leucovorin, and 2,000 mg/m fluorouracil (modified FOLFOX) or $70 \mathrm{mg} / \mathrm{m}^{2}$ irinotecan, $400 \mathrm{mg} / \mathrm{m}^{2}$ leucovorin, and $2,000 \mathrm{mg} / \mathrm{m}^{2}$ fluorouracil (modified FOLFIRI.3) ${ }^{27}$ However, the efficacy was modest, and the median OS was 14.9 and 16.6 weeks for modified FOLFOX and modified FOLFIRI.3, respectively.

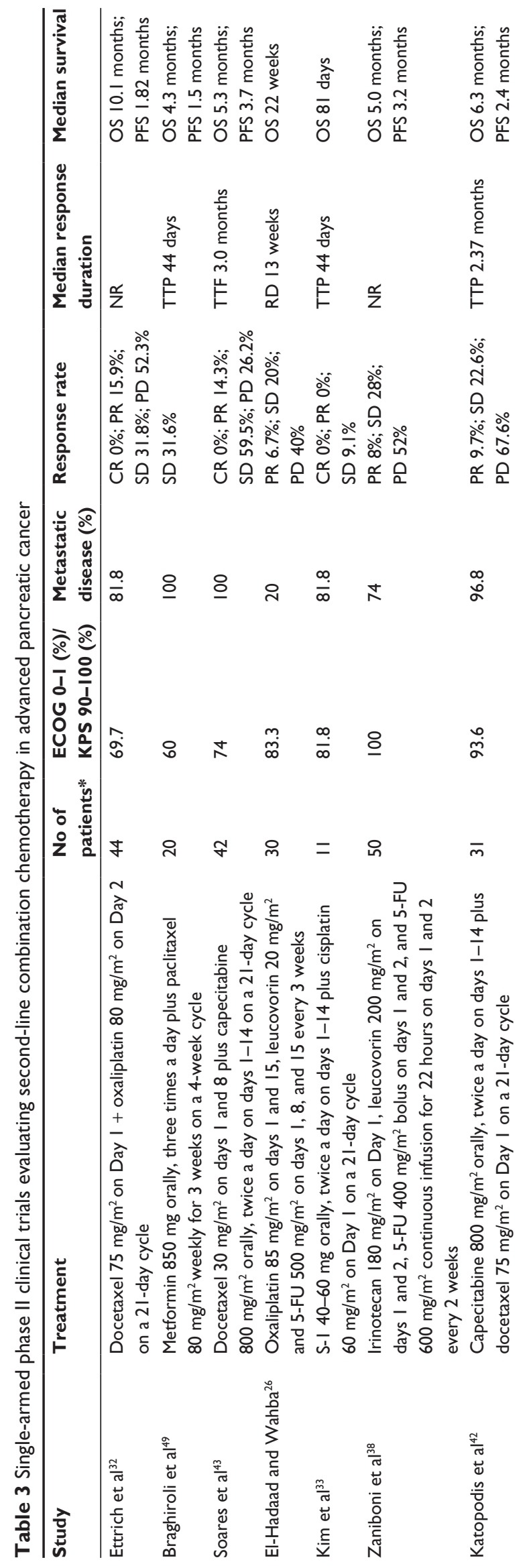




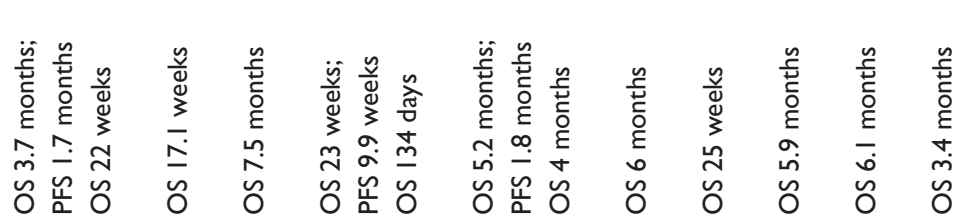

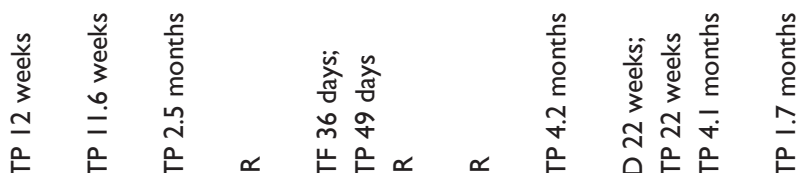

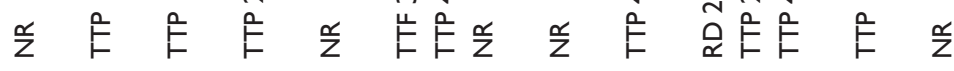

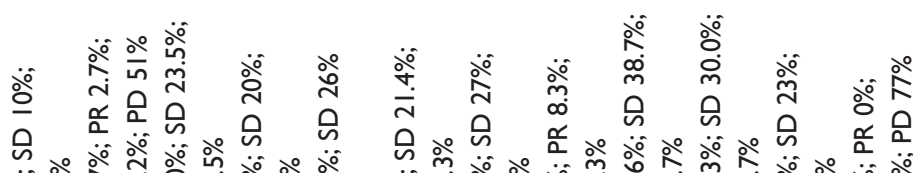

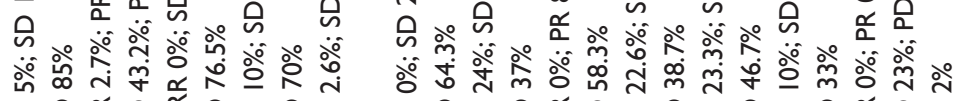

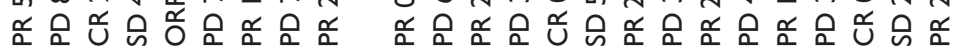

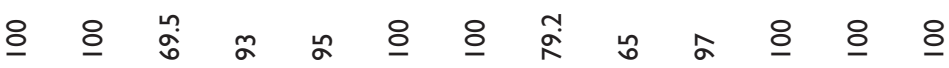

○ 孚

구

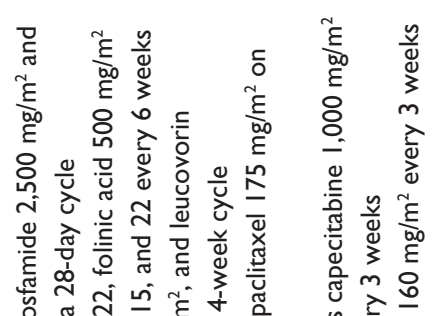

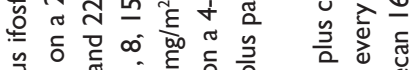

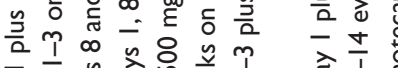

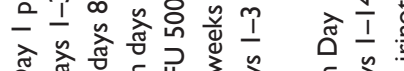

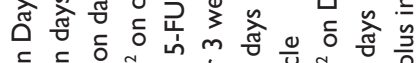

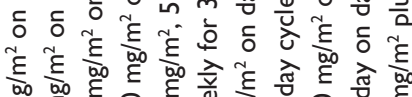

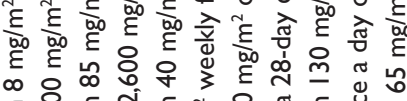

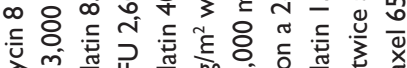

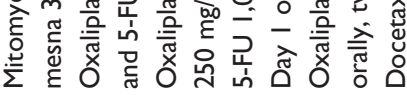

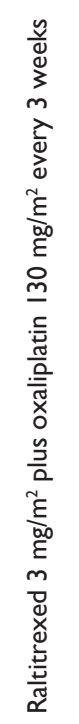

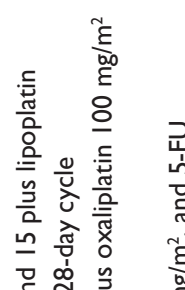

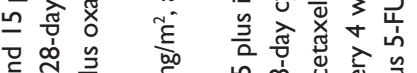

든

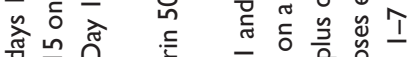

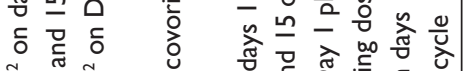

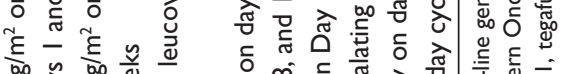

कू 命

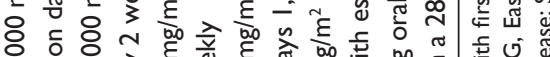

- స

bo

氜

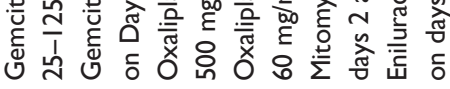

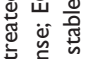

즌

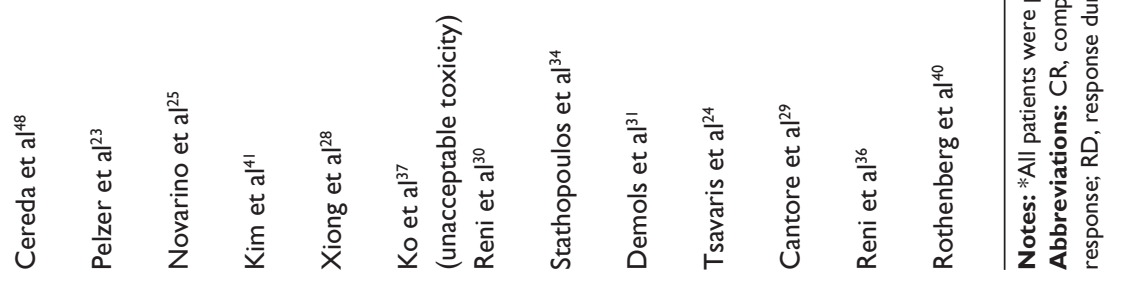


A phase II study assessed the activity and safety profile of a combination of capecitabine and oxaliplatin. ${ }^{28} \mathrm{~A}$ total of 39 patients received this regimen as a second-line treatment. One patient (2.6\%) showed a partial response, and ten patients had stable diseases (26\%). The median PFS and OS were 9.9 and 23 weeks, respectively, and the toxic effects were generally manageable.

Oxaliplatin in combination with agents other than fluoropyrimidine has also been tested as a second-line regimen for advanced pancreatic cancer. A phase II trial evaluated oxaliplatin in combination with irinotecan in 30 patients. ${ }^{29}$ Three patients $(10 \%)$ showed a partial response, and seven patients (23\%) had stable disease. The median TTP was 4.1 months, and the median OS was 5.9 months, with a 1-year survival rate of $23.3 \%$. Grades 3 and 4 adverse events included leukopenia (6\%), neuropathy (6\%), and diarrhea (3\%). Reni et al treated 41 patients, who previously received gemcitabine in the first-line setting, with oxaliplatin at $130 \mathrm{mg} / \mathrm{m}^{2}$ and raltitrexed at $3 \mathrm{mg} / \mathrm{m}^{2}$ every 3 weeks as salvage chemotherapy. ${ }^{30}$ The results showed a partial response in $24 \%$ of patients and a median OS of 5.2 months. Neutropenia was the most common toxic effect, occurring in $12 \%$ of patients. Demols et al evaluated the activity and tolerability of gemcitabine and oxaliplatin (GEMOX) in patients who had progressed during or following gemcitabine therapy. ${ }^{31}$ The study showed a response rate of $22.6 \%$ in 31 assessable patients. The median response duration and TTP were 4.5 and 4.2 months, respectively, and the median OS was 6 months (range $0.5-21$ ). Grades 3 and 4 toxicities occurred in 16 patients (48\%). Recently, Ettrich et al conducted a phase II trial examining the activity of the docetaxel and oxaliplatin (DocOx) combination as a second-line treatment for advanced pancreatic cancer. ${ }^{32}$ Tumor response was achieved in $15.9 \%$ of the patients, with a disease control rate of $48 \%$ after the first two treatment cycles. The median PFS was 1.8 months, and the median OS was 10.1 months. Notably, grades 3 and 4 neutropenia developed in $63.6 \%$ of patients, and diarrhea developed in $11.4 \%$ of patients. However, all toxicities were manageable. Thus, the data obtained using the DocOx protocol were consistent with other second-line protocols, including the OFF regimen, indicating DocOx as an option for patients with chemorefractory pancreatic cancer.

Cisplatin-based regimens have also been investigated as second-line chemotherapy after gemcitabine failure. Kim et al conducted a phase II study examining cisplatin plus S-1 as a second-line palliative chemotherapy for patients with gemcitabine-refractory pancreatic cancer. ${ }^{33}$ The study was terminated early after the enrollment of 11 patients due to severe toxicity. The median TTP was 44 days (1.4 months), and the median OS was 81 days (2.7 months). The most common grades 3 and 4 toxicities included fatigue (27.3\%), nausea $(27.3 \%)$, and anorexia (18.2\%). Stathopoulos et al tested lipoplatin - a liposomal cisplatin aimed at the avoidance of renal toxicity - combined with gemcitabine in pretreated patients with advanced pancreatic cancer. ${ }^{34}$ The objective response rate data showed a partial response in two of the 24 patients examined $(8.3 \%)$ and stable disease in 14 patients $(58.3 \%)$. The median survival from the beginning of second-line treatment was 4 months.

\section{Irinotecan-based regimens}

Irinotecan-based chemotherapeutic regimens are options in the second-line setting for patients who progressed during gemcitabine-based treatment. In Austria, Ulrich-Pur et al conducted a phase II trial involving 38 patients who were pretreated with gemcitabine. ${ }^{35}$ The patients were randomized to 3 -weekly courses of irinotecan at $200 \mathrm{mg} / \mathrm{m}^{2}$ on Day 1 plus raltitrexed at $3 \mathrm{mg} / \mathrm{m}^{2}$ on Day 2 , or raltitrexed at $3 \mathrm{mg} / \mathrm{m}^{2}$ on Day 1 only. The superior response rate in the combination group ( $16 \%$ vs $0 \%)$ led to the termination of the trial in the first stage of accrual. The median PFS (4.0 vs 2.5 months) and the median OS (6.5 vs 4.3 months) were also superior in the combination group. Grades 3 and 4 toxic effects were comparable between the two groups. A study in Italy evaluated the activity of mitomycin, docetaxel, and irinotecan (MDI) on metastatic pancreatic adenocarcinoma following gemcitabine-containing chemotherapy. ${ }^{36}$ The median TTP was 1.7 months, and the median OS was 6.1 months, with no objective response observed. Ko et al tested a regimen using irinotecan and docetaxel and showed a disease control rate of $21.4 \%$. The median OS was 134 days (4.5 months), with a 6-month survival rate of $36 \% .{ }^{37}$ However, 12 of the 14 patients experienced grades 3 and 4 toxic effects, with neutropenia/leukopenia (42.9\%) being the most common grades 3 and 4 toxicity. The FOLFIRI regimen has also been tested in a prospective multicenter study. ${ }^{38}$ Among the 50 enrolled patients, four partial responses (8\%) and 14 stable diseases (28\%) were observed. The median PFS and OS were 3.2 and 5.0 months, respectively. Grades 3 and 4 neutropenia and diarrhea occurred in ten $(20 \%)$ and six (12\%) patients, respectively.

An open-labeled, multicenter, phase III trial (NAPOLI-1) assessed the effect of nanoliposomal irinotecan alone and in combination with FF in patients with metastatic pancreatic ductal adenocarcinoma previously treated with 
gemcitabine-based therapy. ${ }^{11}$ Patients were randomly assigned to receive either $120 \mathrm{mg} / \mathrm{m}^{2}$ nanoliposomal irinotecan every 3 weeks or FF weekly for 4 weeks in a 6-week cycle. Safety data from a concurrent study in patients with metastatic colorectal cancer treated with a combination of $80 \mathrm{mg} / \mathrm{m}^{2}$ nanoliposomal irinotecan and FF every 2 weeks were demonstrated; thus, the study protocol was amended to add this regimen as a third arm. The median OS in patients assigned to the nanoliposomal irinotecan combination regimen was 6.1 months versus 4.2 months with FF alone (HR $=0.67 ; P=0.012$ ). The median OS did not differ between nanoliposomal irinotecan alone and $\mathrm{FF}(\mathrm{HR}=0.99$; $P=0.94)$. The most frequent grades 3 and 4 adverse events in the nanoliposomal irinotecan combination group were neutropenia (27\%), diarrhea (13\%), vomiting (11\%), and fatigue (14\%). The US Food and Drug Administration rapidly approved nanoliposomal irinotecan plus FF as second-line therapy for metastatic pancreatic ductal adenocarcinoma.

\section{Fluorouracil-based regimens}

Fluorouracil in combination with oxaliplatin or irinotecan formulations has been well studied, with the combination of fluorouracil and irinotecan formulations appearing to be appropriate as second-line treatment. ${ }^{39}$ Besides, fluorouracil combined with agents other than oxaliplatin and irinotecan has also been tested. An early phase II trial assessed the combination of eniluracil - a potent irreversible inactivator of dihydropyrimidine dehydrogenase - and fluorouracil. ${ }^{40}$ Among 48 patients with gemcitabine-refractory metastatic pancreatic cancer, one patient ( $2 \%$ ) had a confirmed partial response. The median OS was 3.4 months, and grades 3 and 4 toxicity occurred in 31 out of $48(65 \%)$ patients. The researchers therefore concluded that fluorouracil plus eniluracil has limited activity in patients with advanced pancreatic cancer and is associated with a high frequency of grades 3 and 4 toxic effects. Kim et al studied the combination of fluorouracil and paclitaxel in 28 patients with a history of previous gemcitabine-based chemotherapy, 20 of whom were assessable. ${ }^{41}$ The study showed a partial response rate of $10 \%$ and stable disease in $20 \%$ of patients. The median TTP and OS were 2.5 and 7.6 months, respectively. Furthermore, the combination of capecitabine and docetaxel has been studied because there are preclinical data showing synergy between these agents. A phase II study that enrolled 31 patients showed that the combination of capecitabine with docetaxel was moderately active in pretreated advanced pancreatic cancer with a partial response rate of $9.7 \%$ and stable disease in $22.6 \%$ of patients. ${ }^{42}$ The median
PFS and OS were 2.4 and 6.3 months, respectively. The most common grades 3 and 4 toxic effect was neutropenia (32.2\%). Moreover, the researchers reported that $20 \%$ of patients had pain control, and $38.7 \%$ of patients maintained their body weight. In another phase II study, a similar regimen, but with different dosing, showed a median PFS and OS of 3.7 and 5.3 months, respectively. ${ }^{43} \mathrm{~A}$ total of $14 \%$ of patients showed an objective response, and as many as 59\% of patients had stable disease for two cycles. Treatment was generally well tolerated, but one patient died, with the cause of death being possibly related to treatment.

Based on preclinical data indicating that leucovorin could enhance the efficacy of S-1, Ge et al conducted a phase II trial enrolling 92 patients with gemcitabine-refractory advanced pancreatic cancer. ${ }^{44}$ The patients were randomly assigned to S-1 in combination with leucovorin or S-1 alone. The study showed no significant differences in the median OS (5.5 vs 6.3 months), median PFS (1.9 vs 3.0 months), and overall response rate ( $4.7 \%$ vs $8.3 \%$ ) between the two groups. Grades 3 and 4 toxic effects were significantly higher in the S-1 plus leucovorin group than in the S-1 group. Based on these efficacy data, the researchers concluded that, compared with S-1, S-1 plus leucovorin did not improve survival in this patient population. Another similar study showed a higher disease control rate in the S-1 plus leucovorin group, but comparable OS in the two groups. ${ }^{45}$ Randomized trials from Japan comparing the efficacy and safety of S-1 plus oxaliplatin (or S-1 plus irinotecan) with that of S-1 alone also showed no significant improvement in OS and PFS. ${ }^{46,47}$

\section{Other regimens}

Cereda et al conducted a phase II trial exploring the activity of the combination of mitomycin and ifosfamide as salvage therapy in patients with gemcitabine-resistant metastatic pancreatic cancer. ${ }^{48}$ The study showed a partial response rate of $5 \%$ (one patient) and stable disease in $10 \%$ of patients. However, grades 3 and 4 toxicities, including neutropenia in $80 \%$ of patients, were poorly tolerated, leading to the premature termination of this trial. Braghiroli et al tested the combination of paclitaxel and metformin for patients with gemcitabine-refractory pancreatic cancer based on preclinical data demonstrating synergism in this combination. ${ }^{49}$ The disease control rate was $31.6 \%$ (6 patients), with a median OS and PFS of 128 and 44 days, respectively. Treatment-related grades 3 and 4 toxicities were observed in eight patients (40\%), with the most common toxicity being diarrhea. The study failed to meet its primary endpoint and was, therefore, terminated in the first phase. 


\section{Second-line targeted therapy: going beyond cytotoxic chemotherapy?}

Targeted agents have been extensively assessed in the firstline setting in pancreatic cancer. Disappointingly, multiple studies have failed to show any improvement in survival. One exception is the combination of gemcitabine and the EGFR tyrosine kinase inhibitor (TKI) erlotinib, which gained regulatory approval following a small but statistically significant improvement (10-day) in median survival compared with gemcitabine alone in a large, randomized phase III trial..$^{50}$ Despite the lack of activity of targeted agents in the first-line setting, investigators have tested new drugs or drug combinations to improve survival for patients with advanced pancreatic cancer following first-line therapy (Table 4).

\section{EGFR inhibitors}

Erlotinib - the most studied targeted agent - has been evaluated in the setting of gemcitabine-refractory metastatic pancreatic cancer in patients. A phase II trial of erlotinib dose-escalation to induce rash in non-selected patients with advanced pancreatic cancer showed a correlation between rash and disease control, with $47 \%$ of evaluable patients with grade 2 or 3 rash showing stable disease over 8 weeks versus $9 \%$ of patients with grade 0 or 1 rash $(P=0.017){ }^{51}$ However, there was no difference in survival based on rash, with a median OS of 3.9 months for patients who developed grade 2 or 3 rash versus 3.8 months for patients with grade 0 or 1 rash $(P=0.12)$. In addition, no differences in median TTP by degree of rash were noted $(P=0.25)$. Erlotinib combined with chemotherapeutic drugs or other targeted agents has also been tested in the second-line setting. A phase II trial studied erlotinib in combination with capecitabine in 30 gemcitabine-refractory patients and showed an overall objective radiologic response rate of $10 \%$ and median survival duration of 6.5 months. ${ }^{52}$ In another phase II trial, the safety and efficacy of selumetinib, a MEK $1 / 2$ inhibitor, plus erlotinib in patients with previously treated advanced pancreatic cancer were evaluated on the basis of preclinical evidence of synergistic activity between MEK and EGFR inhibitors. ${ }^{53}$ This dual-targeted therapeutic strategy showed modest antitumor activity in pancreatic cancer, with a disease control rate of $41 \%$ and a median OS of 7.3 months. Dual inhibition of the EGFR and VEGF pathways using the combination of erlotinib and bevacizumab has also been tested for gemcitabine-refractory metastatic pancreatic cancer. ${ }^{54}$ With a median OS of 102 days and median TTP of 40 days, the authors concluded that this "targeted-only" approach was relatively ineffective for this patient population. Everolimus, an mammalian target of rapamycin (mTOR) inhibitor, and erlotinib in combination were also evaluated as another dual-targeted regimen. ${ }^{55}$ The researchers reported a median OS of 87 days (2.9 months), with no objective response or disease stability.

Gefitinib, another EGFR TKI, was shown to enhance the activity of various cytotoxic agents, including taxanes, in preclinical studies. Ignatiadis et al, therefore, conducted a phase II trial using a gefitinib and docetaxel combination as second-line treatment in patients with advanced pancreatic cancer. ${ }^{56}$ The study enrolled 26 patients and showed a median OS of 2.9 months, with $34.6 \%$ of patients developing grade 3/4 neutropenia (only one developed febrile neutropenia). A similar study done by Brell et al also showed limited efficacy. Notably, febrile neutropenia was observed in 11 patients $(27 \%))^{57}$

Lapatinib is a TKI that binds to both EGFR and HER-2, which is overexpressed in $20 \%$ of patients with pancreatic cancer. Lapatinib leads to cell-growth arrest by the dual inhibition of EGFR and HER-2 and apoptosis via the inhibition of HER-2. Based on preclinical data suggesting augmented inhibition of tumor progression by targeting EGFR and HER-2 in xenograft models, Wu et al treated 17 patients with metastatic pancreatic cancer, who had progressed during first-line gemcitabine-based therapy, with capecitabine at $1,000 \mathrm{mg} / \mathrm{m}^{2}$ on days $1-14$ in combination with lapatinib $1,250 \mathrm{mg}$ daily on a 21 -day cycle..$^{58} \mathrm{~A}$ disease control rate of $35.3 \%$ was achieved, with median PFS and OS rates of 2.6 and 5.2 months, respectively. Furthermore, the researchers observed that a subset of patients who responded to the lapatinib and capecitabine showed prolonged PFS (4.0 months) and OS (8.3 months).

\section{VEGF and VEGFR inhibitors}

Bevacizumab - an anti-VEGF monoclonal antibody - has been approved for the treatment of various solid tumors (colon cancer, non-small-cell lung cancer, and renal cancer) and a specific eye disease. A previous phase III trial comparing gemcitabine plus bevacizumab with gemcitabine alone in the first-line setting showed no improvement in OS with the addition of bevacizumab to gemcitabine..$^{59}$ In the second-line setting, patients with gemcitabine-refractory metastatic pancreatic adenocarcinoma were randomized to receive bevacizumab alone or bevacizumab in combination with docetaxel. ${ }^{60}$ The median PFS and OS were 43 days (1.4 months) and 165 days (5.5 months) in the combination group and 48 days (1.6 months) and 125 days (4.2 months) in the single-agent group. No confirmed objective responses 


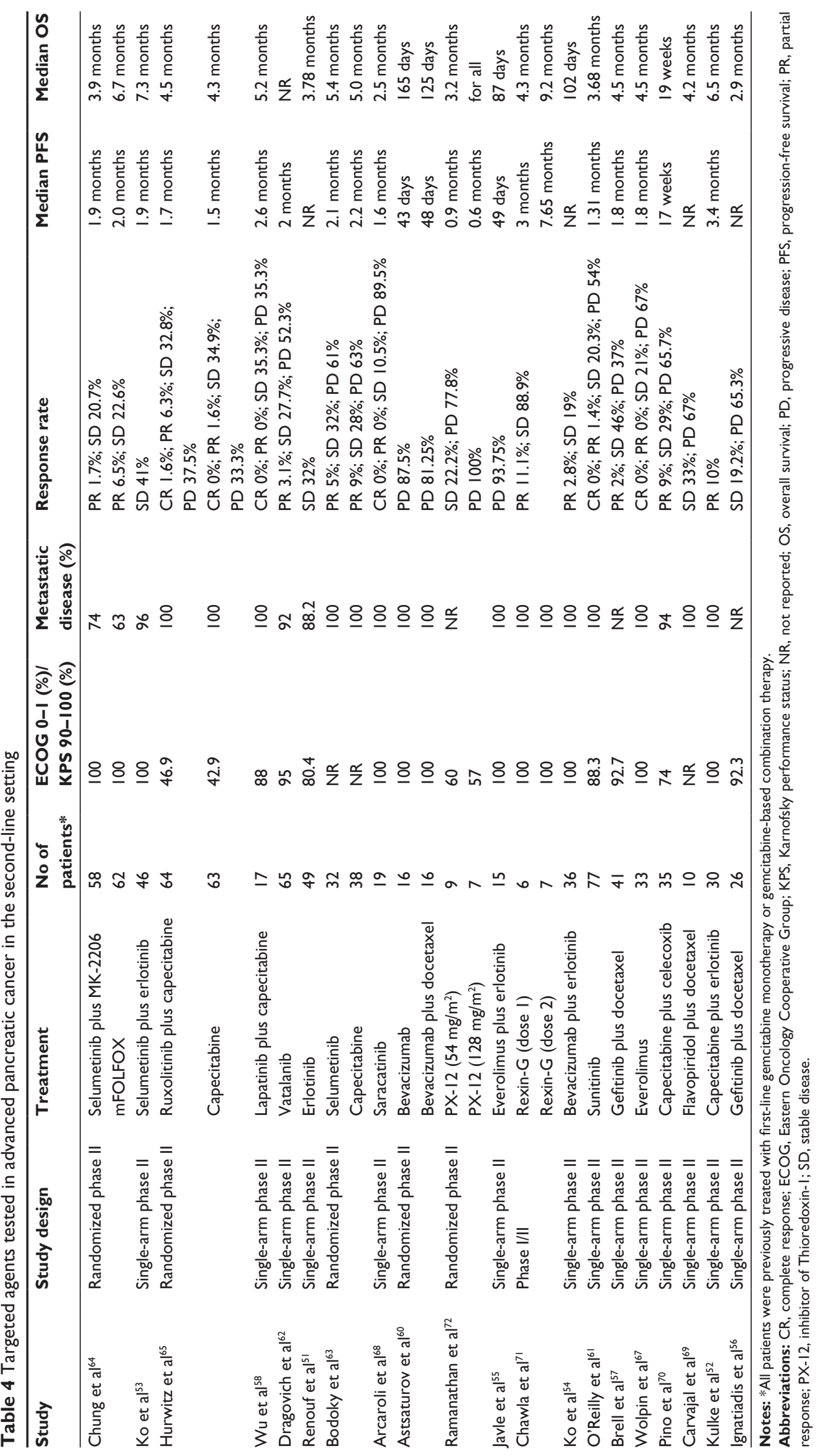


were observed, and the study was terminated according to the early termination rule for futility.

Sunitinib is an oral, small-molecule, multi-targeted receptor TKI that inhibits VEGFR, PDGFR, kit, RET, and FLT3. The Cancer and Leukemia Group B conducted a phase II study evaluating sunitinib in 77 patients with progressive metastatic pancreatic cancer following prior gemcitabinebased therapy. ${ }^{61}$ The disease control rate was $21.7 \%$ : one patient $(1.4 \%)$ had a partial response, and 15 patients had stable disease (20.3\%). The PFS was 1.31 months and OS was 3.68 months. Although the study met its primary endpoint (disease control rate), the researchers concluded that sunitinib had minimal activity and moderate toxicity in a population of patients with gemcitabine-refractory pancreatic cancer.

Vatalanib is an orally active TKI with high receptor-binding affinity for VEGFR, PDGFR, c-Kit, and c-Fms. In preclinical orthotopic pancreatic models, vatalanib showed significant antitumor activity and it was correlated with decreased microvessel density. As vatalanib targets multiple targets implicated in pancreatic cancer survival and angiogenesis and shows promising preclinical activity, a phase II study investigating the efficacy and tolerability of vatalanib in patients with gemcitabine-refractory advanced pancreatic cancer was conducted. ${ }^{62}$ The study resulted in a 6-month survival rate of $29 \%$, with a median PFS of 2 months. The most common grade $3 / 4$ adverse events included hypertension (20\%), fatigue (17\%), abdominal pain $(17 \%)$, and elevated alkaline phosphatase $(15 \%)$. Changes in biomarkers, including soluble VEGF and VEGFR, did not correlate with the response to vatalanib.

\section{Ras pathway inhibitors}

The Ras pathway represents a growth-promoting pathway that is associated with pancreatic cancer through mutations in the $K R A S$ and $B R A F$ genes. Activating $K R A S$ mutations are observed in $>90 \%$ of pancreatic adenocarcinomas. Reflecting the complexity of directly targeting KRAS, drug development efforts have focused on downstream components of the Ras pathway, such as MEK. Selumetinib is an oral MEK1/2 inhibitor, and a free-base suspension of selumetinib has demonstrated preclinical activity in a range of tumors, including human pancreatic tumors. In a randomized phase II study, 70 patients with metastatic pancreatic cancer, who failed first-line therapy, were administered either selumetinib or capecitabine. ${ }^{63}$ The median OS was 5.4 months in the selumetinib group and 5.0 months in the capecitabine group $(P=0.92)$. The median PFS was 2.1 and 2.2 months in the selumetinib and capecitabine groups, respectively $(P=0.41)$. Serious adverse events were recorded in $43 \%$ of patients in the selumetinib group and $25 \%$ in the capecitabine group.
These researchers concluded that although selumetinib is a well-tolerated second-line option for metastatic pancreatic cancer patients with failed first-line gemcitabine treatment, selumetinib is not significantly different from capecitabine in terms of OS in this patient population. Furthermore, the dualtargeted therapeutic strategy using selumetinib and MK-2206 failed to improve OS in the second-line setting. ${ }^{64}$

\section{Janus kinase inhibitors}

Emerging evidence supports a role for Janus kinase/signal transducer and activator of transcription (JAK/STAT) signaling in the pathogenesis and clinical course of pancreatic cancer. In a randomized phase II study, the JAK1/JAK2 inhibitor ruxolitinib was evaluated in combination with capecitabine versus placebo plus capecitabine in patients who experienced treatment failure with gemcitabine. ${ }^{65}$ No difference in survival was observed in the intention-to-treat population. However, in a prespecified subgroup analysis, patients with a C-reactive protein (CRP) level greater than $13 \mathrm{mg} / \mathrm{L}$ showed an improvement in median OS (2.7 vs 1.8 months; $P=0.011$ ). This finding led to the design of two randomized phase III trials (JANUS1 and JANUS2) for the second-line treatment of advanced pancreatic cancer. However, both JANUS1 and JANUS2 studies were terminated due to the lack of efficacy (no survival benefit for ruxolitinib plus capecitabine). ${ }^{66}$

\section{Phosphoinositide-3-kinase (PI3K)-mTOR pathway inhibitors}

The PI3K/Akt/mTOR pathway is activated in half of pancreatic cancers, and inhibition of this pathway has been found to have antitumor effects in preclinical studies. Wolpin et al conducted a phase II trial evaluating the efficacy and safety of everolimus, an oral mTOR inhibitor, in patients who experienced treatment failure on first-line therapy with gemcitabine. ${ }^{67}$ Although treatment with everolimus was well tolerated, no complete or partial responses were observed, and only seven of 33 patients $(21 \%)$ had stable disease at the first restaging scans performed at 2 months. The median PFS and OS was 1.8 and 4.5 months, respectively.

Src, a nonreceptor tyrosine kinase, interacts with the PI3K regulatory subunit $\mathrm{p} 85$ to yield an increase in Akt activation. The oral Src inhibitor saracatinib exhibited antitumor activity in preclinical models of pancreatic cancer. In a phase II clinical trial of saracatinib in patients previously treated for pancreatic cancer, saracatinib was administered orally and continuously in 28-day cycles. ${ }^{68}$ As only two patients $(11 \%)$ patients survived for at least 6 months, the study was amended as a biomarker-driven trial. However, 
of the 47 patients screened, only one patient was biomarker positive. Reflecting the low frequency of biomarker-positive patients, the study was eventually terminated.

Some other targeted agents, including flavopiridol (CDK inhibitor), celecoxib (selective COX-2 inhibitor), Rexin-G (targeted gene therapy vector), and PX-12 (inhibitor of thioredoxin-1), have been tested as either single agents or in combination with cytotoxic therapy in the secondline setting. ${ }^{69-72}$ However, most of these drugs resulted in minimal or modest antitumor activity, except for Rexin-G. As a tumor-targeted retrovector bearing a cytocidal cyclin G1 product, Rexin-G is a paradigm in the length of time to bring new therapeutics to bedside. It is the first targeted gene therapy vector to gain fast-track designation and orphan drug priorities for multiple cancer indications in the USA. As for pancreatic cancer, Rexin-G showed promise in the treatment of gemcitabine-resistant metastatic pancreatic cancer, as it produces an almost 10-month survival benefit. A pivotal phase II/III two-arm randomized study has been planned to confirm the OS benefit of Rexin-G as monotherapy versus the physician's choice of therapy in gemcitabine-refractory pancreatic cancer.

Overall, using single or combination targeted agents in the second-line setting has yielded rare responses, short PFS and OS times, and has limited the value with this strategy. Slightly more activity has been observed for combining a targeted agent with cytotoxic therapy in the refractory disease setting, although the benefit may relate to the cytotoxic backbone. In addition, an assessment of biomarkers that might identify the patient subsets most likely to benefit from targeted therapy is needed.

\section{Treatment effect trends over time}

The above-summarized trials with available data were analyzed for treatment effect trends over time. The outcome trends over time were analyzed through the locally estimated scatterplot smoothing (LOESS) method using SPSS 19.0 software (IBM Corporation, Chicago, IL, USA). The median OS, PFS, and response rates of each regimen were plotted over time based on the year the studies were initiated. Regrettably, the median OS, PFS, and response rates remained unchanged over time (Figure 2).

\section{Future perspectives: every cloud has a silver lining Ongoing trials in the second-line setting: finding diamonds in the rough}

As the search continues for strategies to refine approaches in treating patients with pancreatic cancer, and only a few studies have focused on second-line therapy. This phenomenon is attributed to the poor performance status of the patients and limited survival benefit of testing agents or regimens after progression of first-line therapy. Nonetheless, there are still some novel noncytotoxic agents showing therapeutic potentials in this setting (Table 5).

Acalabrutinib, a selective second-generation BTK inhibitor, showed promising antitumor effects in combination with pembrolizumab. ${ }^{73}$ A phase II study evaluating acalabrutinib alone or in combination with pembrolizumab is currently ongoing. Preliminary results of the first 58 treated patients revealed that the combination of acalabrutinib and pembrolizumab has a favorable benefit/risk profile and encouraging antitumor activity in pretreated metastatic pancreatic cancer, particularly in a subpopulation of patients with familial pancreatic cancer.

ERY-ASP - a new investigational medicinal product composed of Escherichia coli L-asparaginase encapsulated in erythrocytes - has shown potential efficacy in a subgroup of patients with pancreatic cancer with null/low asparagine synthetase (79.4\%). ${ }^{74}$ In an ongoing phase II trial, the combination of ERY-ASP plus cytotoxic chemotherapy versus chemotherapy alone is currently being tested.

Similarly, poly (ADP-ribose) polymerase (PARP) inhibitors also hold promise in the treatment of pancreatic cancer. Several trials investigating PARP inhibitors that include olaparib, veliparib, and rucaparib are underway for patients with pretreated advanced pancreatic cancer. Some have, shown encouraging results in patients with a germline BRCA1/2 mutation.

In immunotherapy studies, AM0010 - a pegylated recombinant human interleukin 10 - has shown evidence of antitumor activity as salvage therapy in metastatic pancreatic adenocarcinoma. In a phase Ib study, AM0010 plus FOLFOX resulted in a median PFS of 3.5 months and a median OS of 10.0 months. The observed immune activation, including clonal T-cell expansion and prolonged objective tumor responses, is encouraging, and this regimen is currently being studied in a phase III trial. Besides, pembrolizumab, a type of immunotherapy that works by blocking the programmed death receptor-1 (PD-1) pathway, has been recommended to treat microsatellite instability-high (MSI-H) or mismatch repair deficient (dMMR) tumors in the second-line setting. ${ }^{75}$

\section{Sequential strategy: improving efficacy of treatment}

To date, the optimum sequential chemotherapy strategy remains an unanswered question in the management of 

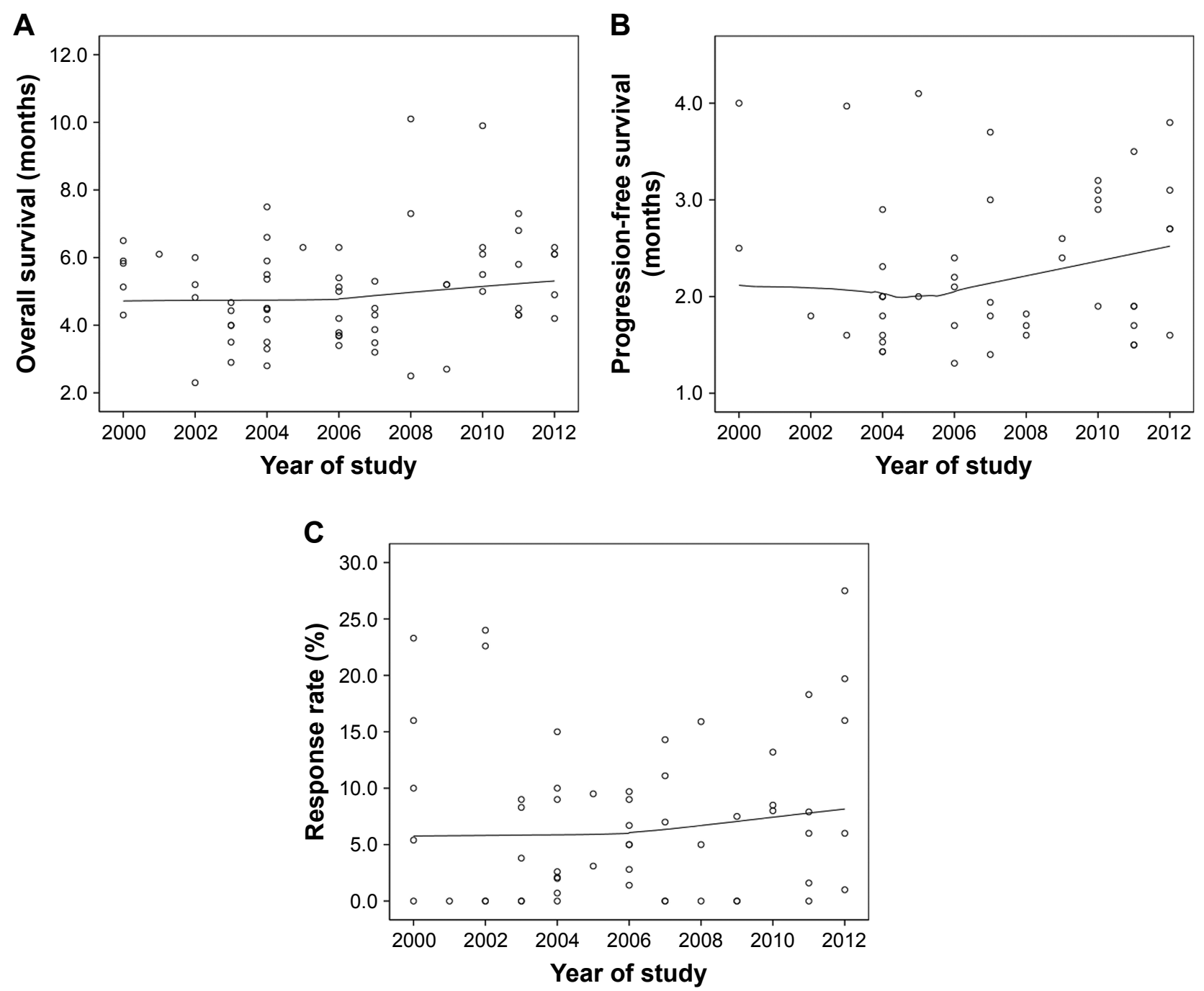

Figure 2 The treatment outcome trends over time plotted against the year of the studies including overall survival (A), progression-free survival (B), and response rate (C).

advanced pancreatic cancer. Because of a lack of relative clinical trials, no clear evidence is available for second-line chemotherapy after FOLFIRINOX or gemcitabine plus nab-paclitaxel first-line regimens. Moreover, the benefit of
FOLFIRINOX, followed by gemcitabine plus nab-paclitaxel versus the reverse sequence, also remains unknown. Recent data from a non-randomized cohort study suggest that the administration of nab-paclitaxel and gemcitabine in patients

Table 5 Selected ongoing phase II or III clinical trials in patients with pretreated advanced pancreatic cancer

\begin{tabular}{lllll}
\hline $\begin{array}{l}\text { Clinical trial } \\
\text { identifier }\end{array}$ & Type of study & Regimen & $\begin{array}{l}\text { No of } \\
\text { patients }\end{array}$ & Primary endpoint \\
\hline NCT02726854 & Phase II & Apatinib & 30 & PFS, ORR \\
NCT02967770 & Randomized phase II & MTT vs SOC & 58 & PFS \\
NCT02558868 & Randomized phase II & Oxaliplatin + irinotecan vs irinotecan & 80 & OS \\
NCT02195I80 & Randomized phase II & ERY-ASP + GEM/FOLFOX4 vs GEM/FOLFOX4 & I4I & PFS \\
NCT02677038 & Phase II & Olaparib & 24 & ORR \\
NCT02042378 & Phase II & Rucaparib & 19 & ORR \\
NCT02890355 & Randomized phase II & Veliparib + mFOLFIRI vs FOLFIRI & OS \\
NCT01954992 & Randomized phase III & Glufosfamide vs 5-FU & 480 & OS \\
NCT0292392I & Randomized phase III & AM00I0 + FOLFOX vs FOLFOX & 566 & OS \\
NCT0I834235 & Randomized phase II & NPC-IC + GEM + ABX vs GEM + ABX & $8 I$ & OS, safety, and tolerability \\
NCT02362048 & Randomized phase II & ACP-I96 vs ACP-I96 + pembrolizumab & 73 & Treatment emergent \\
& & & & adverse events \\
\hline
\end{tabular}

Abbreviations: MTT, molecularly tailored therapy; ORR, objective response rate; OS, overall survival; PFS, progression-free survival; SOC, standard of care; 5-FU, 5-fluorouracil; GEM, gemcitabine; ERY-ASP, L-asparaginase encapsulated in erythrocytes; NPC-IC, ensituximab; ABX, nano albumin bound-paclitaxel; ACP-I96, acalabrutinib; FOLFOX, folinic acid + fluorouracil + oxaliplatin; FOLFIRI, folinic acid + fluorouracil + irinotecan. 
refractory to the FOLFIRINOX regimen might be a successful strategy to delay tumor progression, as documented by a disease control rate of 58\%. ${ }^{76}$ Median PFS and OS from the start of second-line therapy were 5.1 and 8.8 months, respectively. However, these exciting results were associated with a high rate of toxicity; grades 3 and 4 toxicity occurred in $40 \%$ of the patients, mostly neutropenia and neurotoxicity. Moreover, a retrospective study using the same sequence observed similar results. ${ }^{77}$ Therefore, to reduce cumulative toxic effects, optimize dose intensity, and increase efficacy, further investigation in therapeutic sequences is needed.

\section{Maintenance therapy in advanced disease: new life for an old idea}

With the success of more effective regimens in patients with advanced disease, questions concerning how best to manage the treatment-free interval prior to disease progression have been raised. Therapeutic strategies, such as maintenance therapy, may represent potential means to improve clinical outcomes in advanced pancreatic cancer. Although well established for the treatment of certain hematologic malignancies, maintenance therapy has only recently been tested as a treatment paradigm for metastatic pancreatic adenocarcinoma. In the Pancreatic AdenoCarcinoma Trials - 12 (PACT-12) trial, 55 patients without evidence of progression after 6 months of initial chemotherapy (mostly, gemcitabine combinations) were randomized to sunitinib or observation. ${ }^{78}$ Median OS was 9.2 months in the observation group versus 10.6 months in the sunitinib group, which was not statistically significant (HR 0.71; 95\% CI 0.40-1.26; $P=0.11$ ) and precluded strong conclusions; however, the 1- and 2-year survival rates were $35.7 \%$ and $7.1 \%$ in the observation arm and $40.7 \%$ and $22.9 \%$ in the sunitinib arm, suggesting that a subset of patients may benefit from maintenance therapy. Although sunitinib proved inactive in second-line therapy of pancreatic cancer, the intriguing results of the PACT-12 trial suggest that this drug may impact the disease course when administered as maintenance treatment in patients achieving disease control with first-line chemotherapy. Alternatively, it is likely that as more active agents against pancreatic cancer become available, maintenance therapy may potentially achieve even more exciting results.

\section{OS prediction for second-line chemotherapy: better patient selection}

Currently, the vast majority of clinical trials evaluating second-line regimens have used the Eastern Oncology Cooperative Group or Karnofsky performance status score and other "pragmatic parameters" (eg, age, duration of first-line therapy) to select candidates. This selection not only generates considerable heterogeneity in survival in patients who receive second-line chemotherapy between studies but also is unable to determine which patients might benefit from second-line chemotherapy. A retrospective study from the CONKO study group revealed that a prognostic score which included Karnofsky performance status, carbohydrate antigen 19-9 levels at start of second-line therapy, and the duration of first-line therapy was able to identify three subgroups in the second-line setting. ${ }^{79}$ The modified Glasgow Prognostic Score - a systemic inflammation-based prognostic system which incorporated CRP and albumin - has also been shown to be reliable indicator of OS in the setting of secondline therapy. ${ }^{65}$ In a prospective population-based cohort study, Vienot et al developed and validated a prognostic nomogram and score to predict OS in patients with advanced pancreatic cancer who received second-line chemotherapy in routine clinical practice using a broad spectrum of parameters (age, smoking status, liver metastases, performance status, pain, jaundice, ascites, duration of first-line, and type of second-line regimen). ${ }^{80}$ This score classified patients into low-, intermediate-, and high-risk groups with median OS of $11.3,3.6$, and 1.4 months, respectively. Although the study did not evaluate albumin or serum CRP because of the high rate of missing data, this prognostic nomogram and score represents the most comprehensive scoring system reported thus far, which accurately predicts OS prior to the administration of second-line chemotherapy and may help clinicians in their therapeutic decisions. In addition, this tool may be beneficial for better selection of patients for treatment, for stratified random assignment to ensure well-balanced treatment groups, and for the potential optimization of clinical trial design. Furthermore, the development of risk-adapted strategies for second-line management in the future could be considered in the different risk groups identified.

\section{Conclusion}

If disease progression occurs after administration of first-line therapy, survival remains short for patients with pancreatic cancer, and the outcome with second-line chemotherapy remains unsatisfactory, with a low response rate. The current treatment options vary in terms of drugs and dosages with different survival benefits. The only way to move forward in meaningful ways will be to identify new and more effective therapeutic alternatives that emerge from phase I/II clinical trials. Any therapy should be individualized and based on performance status, comorbidities, expected toxicities, and patient preference. More broadly, with a greater understanding of pancreatic cancer tumor biology, coupled with the 
growing availability of non-cytotoxic agents and interest by academia and pharmaceutical companies to meet the challenge, better systemic treatments of pancreatic cancer beyond conventional chemotherapy are anticipated in the near future. Due to the heterogeneity of pancreatic cancer per se, each novel therapy may exclusively be limited to a distinct subset of individuals. In addition, there is a need to assess biomarkers that might identify the patient subsets that will most likely benefit from second-line therapy.

\section{Acknowledgments}

This work was supported by the National Science Foundation for Distinguished Young Scholars of China (grant no 81625016) and the Shanghai Sailing Program (grant no 17YF1402500).

\section{Author contributions}

All authors contributed toward data analysis, drafting and critically revising the paper and agree to be accountable for all aspects of the work.

\section{Disclosure}

The authors report no conflicts of interest in this work.

\section{References}

1. Siegel RL, Miller KD, Jemal A. Cancer statistics, 2015. CA Cancer J Clin. 2015;65(1):5-29.

2. Siegel RL, Miller KD, Jemal A. Cancer statistics, 2016. CA Cancer J Clin. 2016;66(1):7-30.

3. Siegel RL, Miller KD, Jemal A. Cancer statistics, 2017. CA Cancer J Clin. 2017;67(1):7-30.

4. Kamisawa T, Wood LD, Itoi T, Takaori K. Pancreatic cancer. Lancet. 2016;388(10039):73-85.

5. Neuzillet C, Tijeras-Raballand A, Bourget P, et al. State of the art and future directions of pancreatic ductal adenocarcinoma therapy. Pharmacol Ther. 2015;155:80-104.

6. Burris HA 3rd, Moore MJ, Andersen J, et al. Improvements in survival and clinical benefit with gemcitabine as first-line therapy for patients with advanced pancreas cancer: a randomized trial. J Clin Oncol. 1997; 15(6):2403-2413.

7. Conroy T, Desseigne F, Ychou M, et al; Groupe Tumeurs Digestives of Unicancer; PRODIGE Intergroup. FOLFIRINOX versus gemcitabine for metastatic pancreatic cancer. N Engl J Med. 2011;364(19):1817-1825.

8. Von Hoff DD, Ervin T, Arena FP, et al. Increased survival in pancreatic cancer with nabpaclitaxel plus gemcitabine. $N$ Engl J Med. 2013;369(18):1691-1703.

9. Dahan L, Bonnetain F, Ychou M, et al; Fédération Francophone de Cancérologie Digestive. Combination 5-fluorouracil, folinic acid and cisplatin (LV5FU2-CDDP) followed by gemcitabine or the reverse sequence in metastatic pancreatic cancer: final results of a randomised strategic phase III trial (FFCD 0301). Gut. 2010;59(11):1527-1534.

10. Oettle H, Riess H, Stieler JM, et al. Second-line oxaliplatin, folinic acid, and fluorouracil versus folinic acid and fluorouracil alone for gemcitabine-refractory pancreatic cancer: outcomes from the CONKO003 trial. J Clin Oncol. 2014;32(23):2423-2429.

11. Wang-Gillam A, Li CP, Bodoky G, et al; NAPOLI-1 Study Group. Nanoliposomal irinotecan with fluorouracil and folinic acid in metastatic pancreatic cancer after previous gemcitabine-based therapy (NAPOLI-1): a global, randomised, open-label, phase 3 trial. Lancet. 2016;387(10018):545-557.
12. Gill S, Ko YJ, Cripps C, et al. PANCREOX: a Randomized phase III study of fluorouracil/leucovorin with or without oxaliplatin for second-line advanced pancreatic cancer in patients who have received gemcitabinebased chemotherapy. J Clin Oncol. 2016;34(32):3914-3920.

13. Ciuleanu TE, Pavlovsky AV, Bodoky G, et al. A randomised Phase III trial of glufosfamide compared with best supportive care in metastatic pancreatic adenocarcinoma previously treated with gemcitabine. Eur J Cancer. 2009;45(9):1589-1596.

14. Pelzer U, Schwaner I, Stieler J, et al. Best supportive care (BSC) versus oxaliplatin, folinic acid and 5-fluorouracil (OFF) plus BSC in patients for second-line advanced pancreatic cancer: a phase III-study from the German CONKO-study group. Eur J Cancer. 2011;47(11):1676-1681.

15. Androulakis N, Syrigos K, Polyzos A, et al; Hellenic Oncology Research Group. Oxaliplatin for pretreated patients with advanced or metastatic pancreatic cancer: a multicenter phase II study. Cancer Invest. 2005;23(1):9-12.

16. Boeck S, Weigang-Köhler K, Fuchs M, et al. Second-line chemotherapy with pemetrexed after gemcitabine failure in patients with advanced pancreatic cancer: a multicenter phase II trial. Ann Oncol. 2007;18(4):745-751.

17. Morizane C, Okusaka T, Furuse J, et al. A phase II study of S-1 in gemcitabine-refractory metastatic pancreatic cancer. Cancer Chemother Pharmacol. 2009;63(2):313-319.

18. Sudo K, Yamaguchi T, Nakamura K, et al. Phase II study of S-1 in patients with gemcitabine-resistant advanced pancreatic cancer. Cancer Chemother Pharmacol. 2011;67(2):249-254.

19. Yi SY, Park YS, Kim HS, et al. Irinotecan monotherapy as second-line treatment in advanced pancreatic cancer. Cancer Chemother Pharmacol. 2009;63(6):1141-1145.

20. Ko AH, Tempero MA, Shan YS, et al. A multinational phase 2 study of nanoliposomal irinotecan sucrosofate (PEP02, MM-398) for patients with gemcitabine-refractory metastatic pancreatic cancer. Br J Cancer. 2013; 109(4):920-925.

21. Boeck S, Wilkowski R, Bruns CJ, et al. Oral capecitabine in gemcitabinepretreated patients with advanced pancreatic cancer. Oncology. 2007; 73(3-4):221-227.

22. Hosein PJ, de Lima Lopes G Jr, Pastorini VH, et al. A phase II trial of nab-Paclitaxel as second-line therapy in patients with advanced pancreatic cancer. Am J Clin Oncol. 2013;36(2):151-156.

23. Pelzer U, Stieler J, Roll L, et al. Second-line therapy in refractory pancreatic cancer. results of a phase II study. Onkologie. 2009;32(3):99-102.

24. Tsavaris N, Kosmas C, Skopelitis H, et al. Second-line treatment with oxaliplatin, leucovorin and 5-fluorouracil in gemcitabine-pretreated advanced pancreatic cancer: a phase II study. Invest New Drugs. 2005;23(4): 369-375.

25. Novarino A, Satolli MA, Chiappino I, et al. Oxaliplatin, 5-fluorouracil, and leucovorin as second-line treatment for advanced pancreatic cancer. Am J Clin Oncol. 2009;32(1):44-48.

26. El-Hadaad HA, Wahba HA. Oxaliplatin plus 5-fluorouracil and folinic acid (OFF) in gemcitabine-pretreated advanced pancreatic cancer: a phase II study. J Gastrointest Cancer. 2013;44(3):313-317.

27. Yoo C, Hwang JY, Kim JE, et al. A randomised phase II study of modified FOLFIRI.3 vs modified FOLFOX as second-line therapy in patients with gemcitabine-refractory advanced pancreatic cancer. $\mathrm{Br} J$ Cancer. 2009;101(10):1658-1663.

28. Xiong HQ, Varadhachary GR, Blais JC, Hess KR, Abbruzzese JL, Wolff RA. Phase 2 trial of oxaliplatin plus capecitabine (XELOX) as second-line therapy for patients with advanced pancreatic cancer. Cancer. 2008;113(8):2046-2052.

29. Cantore M, Rabbi C, Fiorentini G, et al. Combined irinotecan and oxaliplatin in patients with advanced pre-treated pancreatic cancer. Oncology. 2004;67(2):93-97.

30. Reni M, Pasetto L, Aprile G, et al. Raltitrexed-eloxatin salvage chemotherapy in gemcitabine-resistant metastatic pancreatic cancer. $\mathrm{Br} J$ Cancer. 2006;94(6):785-791.

31. Demols A, Peeters M, Polus M, et al. Gemcitabine and oxaliplatin (GEMOX) in gemcitabine refractory advanced pancreatic adenocarcinoma: a phase II study. Br J Cancer. 2006;94(4):481-485. 
32. Ettrich TJ, Perkhofer L, von Wichert G, et al. DocOx (AIO-PK0106): a phase II trial of docetaxel and oxaliplatin as a second line systemic therapy in patients with advanced pancreatic ductal adenocarcinoma. BMC Cancer. 2016;16:21.

33. Kim HJ, Yun J, Kim HJ, et al. Phase II study of palliative S-1 in combination with cisplatin as second-line chemotherapy for gemcitabinerefractory pancreatic cancer patients. Oncol Lett. 2012;3(6):1314-1318.

34. Stathopoulos GP, Boulikas T, Vougiouka M, Rigatos SK, Stathopoulos JG. Liposomal cisplatin combined with gemcitabine in pretreated advanced pancreatic cancer patients: a phase I-II study. Oncol Rep. 2006;15(5):1201-1204.

35. Ulrich-Pur H, Raderer M, Verena Kornek G, et al. Irinotecan plus raltitrexed vs raltitrexed alone in patients with gemcitabine-pretreated advanced pancreatic adenocarcinoma. Br J Cancer. 2003;88(8):1180-1184.

36. Reni M, Panucci MG, Passoni P, et al. Salvage chemotherapy with mitomycin, docetaxel, and irinotecan (MDI regimen) in metastatic pancreatic adenocarcinoma: a phase I and II trial. Cancer Invest. 2004;22(5):688-696

37. Ko AH, Dito E, Schillinger B, Venook AP, Bergsland EK, Tempero MA. Excess toxicity associated with docetaxel and irinotecan in patients with metastatic, gemcitabine-refractory pancreatic cancer: results of a phase II study. Cancer Invest. 2008;26(1):47-52.

38. Zaniboni A, Aitini E, Barni S, et al. FOLFIRI as second-line chemotherapy for advanced pancreatic cancer: a GISCAD multicenter phase II study. Cancer Chemother Pharmacol. 2012;69(6):1641-1645.

39. Sonbol MB, Firwana B, Wang Z, et al. Second-line treatment in patients with pancreatic ductal adenocarcinoma: a meta-analysis. Cancer. 2017;123(23):4680-4686.

40. Rothenberg ML, Benedetti JK, Macdonald JS, et al. Phase II trial of 5-fluorouracil plus eniluracil in patients with advanced pancreatic cancer: a Southwest Oncology Group study. Ann Oncol. 2002;13(10):1576-1582.

41. Kim YJ, Bang S, Park JY, Park SW, Chung JB, Song SY. Phase II study of 5-fluorouracil and paclitaxel in patients with gemcitabine-refractory pancreatic cancer. Cancer Chemother Pharmacol. 2009;63(3):529-533.

42. Katopodis O, Polyzos A, Kentepozidis N, et al. Second-line chemotherapy with capecitabine (Xeloda) and docetaxel (Taxotere) in previously treated, unresectable adenocarcinoma of pancreas: the final results of a phase II trial. Cancer Chemother Pharmacol. 2011;67(2):361-368.

43. Soares HP, Bayraktar S, Blaya M, et al. A phase II study of capecitabine plus docetaxel in gemcitabine-pretreated metastatic pancreatic cancer patients: CapTere. Cancer Chemother Pharmacol. 2014;73(4):839-845.

44. Ge F, Xu N, Bai Y, et al. S-1 as monotherapy or in combination with leucovorin as second-line treatment in gemcitabine-refractory advanced pancreatic cancer: a randomized, open-label, multicenter, phase II study. Oncologist. 2014;19(11):1133-1134.

45. Ueno M, Okusaka T, Omuro Y, et al. A randomized phase II study of S-1 plus oral leucovorin versus S-1 monotherapy in patients with gemcitabine-refractory advanced pancreatic cancer. Ann Oncol. 2016;27(3):502-508.

46. Ohkawa S, Okusaka T, Isayama H, et al. Randomised phase II trial of S-1 plus oxaliplatin vs S-1 in patients with gemcitabine-refractory pancreatic cancer. Br J Cancer. 2015;112(9):1428-1434.

47. Ioka T, Komatsu Y, Mizuno N, et al. Randomized phase II trial of irinotecan plus S-1 in patients with gemcitabine-refractory pancreatic cancer. Br J Cancer. 2017;116(4):464-471.

48. Cereda S, Reni M, Rognone A, et al. Salvage therapy with mitomycin and ifosfamide in patients with gemcitabine-resistant metastatic pancreatic cancer: a phase II trial. Chemotherapy. 2011;57(2):156-161.

49. Braghiroli MI, de Celis Ferrari AC, Pfiffer TE, et al. Phase II trial of metformin and paclitaxel for patients with gemcitabine-refractory advanced adenocarcinoma of the pancreas. Ecancermedicalscience. 2015;9:563.

50. Moore MJ, Goldstein D, Hamm J, et al; National Cancer Institute of Canada Clinical Trials Group. Erlotinib plus gemcitabine compared with gemcitabine alone in patients with advanced pancreatic cancer: a phase III trial of the National Cancer Institute of Canada Clinical Trials Group. J Clin Oncol. 2007;25(15):1960-1966.
51. Renouf DJ, Tang PA, Hedley D, et al. A phase II study of erlotinib in gemcitabine refractory advanced pancreatic cancer. Eur J Cancer. 2014;50(11):1909-1915.

52. Kulke MH, Blaszkowsky LS, Ryan DP, et al. Capecitabine plus erlotinib in gemcitabine-refractory advanced pancreatic cancer. J Clin Oncol. 2007;25(30):4787-4792.

53. Ko AH, Bekaii-Saab T, Van Ziffle J, et al. A multicenter, open-label phase II clinical trial of combined MEK plus EGFR inhibition for chemotherapy-refractory advanced pancreatic adenocarcinoma. Clin Cancer Res. 2016;22(1):61-68.

54. Ko AH, Venook AP, Bergsland EK, et al. A phase II study of bevacizumab plus erlotinib for gemcitabine-refractory metastatic pancreatic cancer. Cancer Chemother Pharmacol. 2010;66(6):1051-1057.

55. Javle MM, Shroff RT, Xiong H, et al. Inhibition of the mammalian target of rapamycin (mTOR) in advanced pancreatic cancer: results of two phase II studies. BMC Cancer. 2010;10:368.

56. Ignatiadis M, Polyzos A, Stathopoulos GP, et al. A multicenter phase II study of docetaxel in combination with gefitinib in gemcitabinepretreated patients with advanced/metastatic pancreatic cancer. Oncology. 2006;71(3-4):159-163.

57. Brell JM, Matin K, Evans T, et al. Phase II study of docetaxel and gefitinib as second-line therapy in gemcitabine pretreated patients with advanced pancreatic cancer. Oncology. 2009;76(4):270-274.

58. Wu Z, Gabrielson A, Hwang JJ, et al. Phase II study of lapatinib and capecitabine in second-line treatment for metastatic pancreatic cancer. Cancer Chemother Pharmacol. 2015;76(6):1309-1314.

59. Van Cutsem E, Vervenne WL, Bennouna J, et al. Phase III trial of bevacizumab in combination with gemcitabine and erlotinib in patients with metastatic pancreatic cancer. J Clin Oncol. 2009;27(13):2231-2237.

60. Astsaturov IA, Meropol NJ, Alpaugh RK, et al. Phase II and coagulation cascade biomarker study of bevacizumab with or without docetaxel in patients with previously treated metastatic pancreatic adenocarcinoma. Am J Clin Oncol. 2011;34(1):70-75.

61. O'Reilly EM, Niedzwiecki D, Hall M, et al; Cancer and Leukemia Group B. A Cancer and Leukemia Group B phase II study of sunitinib malate in patients with previously treated metastatic pancreatic adenocarcinoma (CALGB 80603). Oncologist. 2010;15(12):1310-1319.

62. Dragovich T, Laheru D, Dayyani F, et al. Phase II trial of vatalanib in patients with advanced or metastatic pancreatic adenocarcinoma after first-line gemcitabine therapy (PCRT O4-001). Cancer Chemother Pharmacol. 2014;74(2):379-387.

63. Bodoky G, Timcheva C, Spigel DR, et al. A phase II open-label randomized study to assess the efficacy and safety of selumetinib (AZD6244 [ARRY-142886]) versus capecitabine in patients with advanced or metastatic pancreatic cancer who have failed first-line gemcitabine therapy. Invest New Drugs. 2012;30(3):1216-1223.

64. Chung V, McDonough S, Philip PA, et al. Effect of selumetinib and MK-2206 vs oxaliplatin and fluorouracil in patients with metastatic pancreatic cancer after prior therapy: SWOG S1115 study randomized clinical trial. JAMA Oncol. 2017;3(4):516-522.

65. Hurwitz HI, Uppal N, Wagner SA, et al. Randomized, double-blind, phase II study of ruxolitinib or placebo in combination with capecitabine in patients with metastatic pancreatic cancer for whom therapy with gemcitabine has failed. J Clin Oncol. 2015;33(34):4039-4047.

66. Hurwitz H, Van Cutsem E, Bendell J, et al. Ruxolitinib + capecitabine in advanced/metastatic pancreatic cancer after disease progression/ intolerance to first-line therapy: JANUS 1 and 2 randomized phase III studies. Invest New Drugs. Epub 2018 Mar 6.

67. Wolpin BM, Hezel AF, Abrams T, et al. Oral mTOR inhibitor everolimus in patients with gemcitabine-refractory metastatic pancreatic cancer. J Clin Oncol. 2009;27(2):193-198.

68. Arcaroli J, Quackenbush K, Dasari A, et al. Biomarker-driven trial in metastatic pancreas cancer: feasibility in a multicenter study of saracatinib, an oral Src inhibitor, in previously treated pancreatic cancer. Cancer Med. 2012;1(2):207-217.

69. Carvajal RD, Tse A, Shah MA, et al. A phase II study of flavopiridol (Alvocidib) in combination with docetaxel in refractory, metastatic pancreatic cancer. Pancreatology. 2009;9(4):404-409. 
70. Pino MS, Milella M, Gelibter A, et al. Capecitabine and celecoxib as second-line treatment of advanced pancreatic and biliary tract cancers. Oncology. 2009;76(4):254-261.

71. Chawla SP, Chua VS, Fernandez L, et al. Advanced phase I/II studies of targeted gene delivery in vivo: intravenous Rexin-G for gemcitabineresistant metastatic pancreatic cancer. Mol Ther. 2010;18(2):435-441.

72. Ramanathan RK, Abbruzzese J, Dragovich T, et al. A randomized phase II study of PX-12, an inhibitor of thioredoxin in patients with advanced cancer of the pancreas following progression after a gemcitabine-containing combination. Cancer Chemother Pharmacol. 2011;67(3):503-509.

73. Wu J, Zhang M, Liu D. Acalabrutinib (ACP-196): a selective secondgeneration BTK inhibitor. J Hematol Oncol. 2016;9:21.

74. Bachet JB, Gay F, Maréchal R, et al. Asparagine synthetase expression and phase I study with L-asparaginase encapsulated in red blood cells in patients with pancreatic adenocarcinoma. Pancreas. 2015; 44(7):1141-1147.

75. National Comprehensive Cancer Network. NCCN clinical practice guidelines in oncology: pancreatic adenocarcinoma, V.3.2017. Available from: https:/www.nccn.org/professionals/physician_gls/pdf/ pancreatic.pdf. Accessed December 10, 2017.
76. Portal A, Pernot S, Tougeron D, et al. Nab-paclitaxel plus gemcitabine for metastatic pancreatic adenocarcinoma after Folfirinox failure: an AGEO prospective multicentre cohort. Br J Cancer. 2015;113(7): 989-995.

77. Zhang Y, Hochster H, Stein S, Lacy J. Gemcitabine plus nab-paclitaxel for advanced pancreatic cancer after first-line FOLFIRINOX: single institution retrospective review of efficacy and toxicity. Exp Hematol Oncol. 2015;4:29.

78. Reni M, Cereda S, Milella M, et al. Maintenance sunitinib or observation in metastatic pancreatic adenocarcinoma: a phase II randomised trial. Eur J Cancer. 2013;49(17):3609-3615.

79. Sinn M, Dälken L, Striefler JK, et al. Second-line treatment in pancreatic cancer patients: who profits? - Results from the CONKO study group. Pancreas. 2016;45(4):601-605.

80. Vienot A, Beinse $\mathrm{G}$, Louvet $\mathrm{C}$, et al. Overall survival prediction and usefulness of second-line chemotherapy in advanced pancreatic adenocarcinoma. J Natl Cancer Inst. 2017;109(10).
OncoTargets and Therapy

\section{Publish your work in this journal}

OncoTargets and Therapy is an international, peer-reviewed, open access journal focusing on the pathological basis of all cancers, potential targets for therapy and treatment protocols employed to improve the management of cancer patients. The journal also focuses on the impact of management programs and new therapeutic agents and protocols on

\section{Dovepress}

patient perspectives such as quality of life, adherence and satisfaction. The manuscript management system is completely online and includes a very quick and fair peer-review system, which is all easy to use. Visit http://www.dovepress.com/testimonials.php to read real quotes from published authors. 